Cahiers $d u$ MONDE RUSSE

\section{Cahiers du monde russe}

Russie - Empire russe - Union soviétique et États indépendants

$51 / 4 \mid 2010$

Sciences humaines et sociales en Russie à l'Âge d'argent

\title{
Irina Paperno, Stories of the Soviet Experience
}

\section{Gábor T. Rittersporn}

\section{(2) OpenEdition}

Journals

Édition électronique

URL : https://journals.openedition.org/monderusse/7392

DOI : 10.4000/monderusse.7392

ISSN : $1777-5388$

Éditeur

Éditions de l'EHESS

Édition imprimée

Date de publication : 25 novembre 2010

Pagination : 719-723

ISBN : 978-2-7132-2316-7

ISSN : $1252-6576$

Référence électronique

Gábor T. Rittersporn, «Irina Paperno, Stories of the Soviet Experience », Cahiers du monde russe [En ligne], 51/4 | 2010, mis en ligne le 09 décembre 2011, consulté le 03 septembre 2022. URL : http:// journals.openedition.org/monderusse/7392; DOI : https://doi.org/10.4000/monderusse.7392

Ce document a été généré automatiquement le 3 septembre 2022

Tous droits réservés 


\title{
Irina Paperno, Stories of the Soviet Experience
}

\author{
Gábor T. Rittersporn
}

\section{RÉFÉRENCE}

Irina PAPERNO, Stories of the Soviet Experience. Memoirs, Diaries, Dreams. Ithaca -

Londres : Cornell University Press, 2009, 285 p.

1 Tout le monde ne ressent pas le besoin de confier au papier son expérience. Ceux qui le mettent en pratique le font parfois uniquement pour se souvenir d'événements et d'impressions qui ne regardent qu'eux-mêmes. Mais, souvent, les chroniqueurs de leur quotidien et les annalistes de leur propre passé sont persuadés que leur vécu réserve des leçons précieuses pour les autres. Irina Paperno a fouillé une centaine de journaux intimes et de mémoires d'auteurs qui écrivaient bel et bien pour des lecteurs. Ces textes ont été publiés à la fin de l'époque soviétique ou juste après. Ce qui intéresse l'auteur porte essentiellement sur une période qui va du début des années 1930 jusqu'à l'aboutissement de l'aventure soviétique.

2 Cette expérience est marquée par l'angoisse, par la peur - inséparables du vécu des diaristes et des mémorialistes, dont l'anxiété n'a pas vraiment disparu avec les temps de terreur ; une expérience qui a poursuivi les auteurs bien au-delà de l'époque où elle a pris corps et que l'on croirait révolue depuis longtemps, mais qui refait surface dans nombre de rêves qu'ils ont décrits des décennies plus tard. Il arrive même que des individus qui ont grandi, ou sont même nés après ces années de terreur, évoquent dans leurs écrits intimes les peurs qu'elles suscitent.

3 Paperno fait un excellent usage des sources. Cette expérimentation de la peur n'est qu'un des fils conducteurs de l'ouvrage. La recherche met en évidence l'autoperception des diaristes, la place qu'ils croyaient occuper dans le paysage soviétique - et qu'ils occupent effectivement dans le contexte postsoviétique - ainsi que les contraintes qui les façonnaient avec presque autant de force que l'angoisse. Dès lors, l'auteur se fait 
historienne, philologue, critique littéraire, ethnographe, elle pratique la psychologie sociale, l'anthropologie historique, l'archéologie du quotidien et l'interprétation des cultures. Cela relèverait d'une véritable gageure que de classer son travail parmi les disciplines académiques dont la définition étriquée fait le bonheur des concepteurs de formulaires!

4 À une exception près, les sources de Paperno proviennent d'intellectuels : écrivains, artistes, universitaires et savants, qui se considéraient comme héritiers de l'intelligentsia insoumise du XIXe siècle. L'auteur montre que l'épreuve de la peur n'a pas été leur seul dénominateur commun. La vision qu'ils avaient de ce destin partagé cimentant leur communauté, du témoignage qu'ils en apportaient, de la place qu'il leur conférait dans l'histoire et de l'importance de chaque personne en tant que dépositaire d'une mémoire, constituait un lien entre personnes qui se reconnaissaient en tant que collectif. Paperno ne sous-estime pas les malheurs qui ont marqué leur vie, mais elle s'attache surtout à explorer les conventions littéraires qu'ils ont reprises pour leur compte, une imagerie téléologique qu'ils partageaient du sens de l'histoire, ainsi que leur embarras après la chute de l'ancien régime dont l'effondrement les a placés devant un futur qu'ils ont quelque difficulté à envisager.

5 Paperno ne fait pas référence à l'idée de Karl Löwith selon laquelle toute conception de l'histoire présuppose le principe du salut. Mais elle creuse dans la même direction en arguant que l'intelligentsia soviétique - ou ce qu'il en reste - demeure confondue depuis qu'elle n'est plus capable de se concevoir comme l'artisan d'une fin heureuse de l'histoire. Le monde postsoviétique n'incarne certes pas les valeurs pour lesquelles elle a souffert. Il n'en reste pas moins que les architectes de la nouvelle Russie se réclament des mêmes valeurs, qui se révèlent incomparablement plus ambiguës que l'intellectuel engagé ne pouvait le croire il y a une vingtaine d'années. De plus, ces valeurs peuvent déboucher sur des pratiques inacceptables pour les légataires de la tradition dissidente et cohabiter sans problème avec des normes que l'intelligentsia des temps soviétiques voyait comme incompatibles avec ses convictions.

6 Dans ces conditions, la production et la publication d'ego-documents relèvent d'une opération de sauvetage. Il ne s'agit pas seulement de sauvegarder une mémoire qui peut disparaître avec les témoins, mais également de préserver le sens du vécu d'une communauté d'aspirants-sauveteurs dans une société où le salut qu'elle voulait représenter est loin de se profiler à l'horizon, et de conserver ne serait-ce que les traces de cette communauté ainsi que de l'autoreprésentation qu'elle a d'elle-même.

7 En s'aventurant plus loin, Paperno interprète deux textes. Au premier abord, ceux-ci viennent de deux galaxies séparées par des années-lumière. L'un est bien connu : il s'agit du journal de bord que l'écrivain Lidija Čukovskaja a tenu pendant des décennies tout au long de ses rencontres et conversations avec Anna Ahmatova, ainsi que de celles dont elle a été témoin en sa compagnie. L'autre consiste en les mémoires d'une ouvrière d'origine paysanne et il s'agit d'un récit qui se transforme en journal intime au fil des années. L'auteur ne maîtrise guère l'art de l'orthographe et son style rend la lecture ici et là assez ardue. Mais celle-ci n'en est pas moins passionnante.

Le journal de Čukovskaja rappelle celui de Johann Peter Eckermann où celui-ci a noté les conversations du conseiller privé du prince Karl August, souverain de Saxe-Weimar et d'Eisenach. Tout comme le compagnon de Goethe, du roi poète et grand charmeur, Čukovskaja a tenté de reproduire le plus fidèlement possible les paroles du monstre sacré de la littérature russe. Tous deux ont décrit au mieux l'environnement et les conditions 
de vie de leur héros, dépeint leurs interlocuteurs et retracé leur propre quotidien. On imagine aisément comme le protagoniste d'Eckermann abordait facilement le thème des arts, des sciences et de différents événements historiques, mais comme il était moins prolixe sur le politique. Il est plus ardu d'appréhender la pagaille déprimante des appartements communautaires où la poétesse et son amie parlaient beaucoup de littérature et d'hommes de lettres, mais où elles devaient chuchoter en évoquant la politique, ce qui arrivait souvent. Si Eckermann n'avait qu'admiration pour l'homme fascinant auquel il était totalement dévoué, Čukovskaja, tout en restant longtemps aveugle, s'est lentement affranchie du culte qu'elle vouait à cette femme envoûtante, ce qui n'a pas entamé son attachement et son immense respect pour son œuvre.

9 Les deux femmes n'étaient pas liées que par la littérature : elles partageaient l'angoisse des années de terreur et la peur pour le fils d'Ahmatova ainsi que pour le mari de Čukovskaja, tous deux arrêtés. Elles ne savaient pas encore, en faisant la queue devant une prison pour avoir des nouvelles - cette même queue qui apparaît dans le Requiem de la poétesse -, que l'époux avait été fusillé. À bien des égards, leur expérience de la peur était un prisme qui filtrait tout ce que la conscience et l'inconscient peuvent refléter. Ce filtre modifiait l'image du passé et du présent, désaxant toute perspective. Une vingtaine d'années plus tard, l'angoisse du passé planait encore sur l'univers des deux femmes. Les compagnes d'infortune n'avaient aucun doute quant à l'identité des instigateurs et des bourreaux, mais elles s'interrogeaient sur la culpabilité du reste de la société.

Eckermann ne devait pas, semble-t-il, trop s'attarder sur le petit monde où Goethe avait tout le loisir de créer une grande œuvre littéraire et d'étudier géologie, minéralogie, botanique, théorie des couleurs et influence de ces dernières sur la psyché... Quant à Čukovskaja, elle n'avait certainement pas l'intention d'écrire une étude ethnographique, mais elle était obligée de mentionner les conditions de vie dans lesquelles son hérö̈ne manquait parfois de tout et devait vivre dans l'enfer créé, le plus souvent à leur insu, par des «autres» dont Sartre n'avait certainement pas idée en écrivant sa fameuse pièce. Malgré tout, l'enfermement asphyxiant de ses personnages condamnés à coexister et les limites étouffantes que leur être imposait à leurs semblables ne sont pas étrangers à l'expérience de la poétesse, de sa chroniqueuse ainsi que d'autres diaristes et mémorialistes de Paperno.

11 L'analyse brillante de ces écrits intimes montre davantage que la captivité dans les appartements communautaires ou les diverses formes de dépendance provoquées par la pénurie d'objets de première nécessité et même d'aliments. Paperno saisit également la pression de codes de comportement imposés par ses personnages eux-mêmes, de jeux de rôle stéréotypés et de gestes symboliques, inséparables du statut et de l'autoperception d'intellectuels qui se respectent. Rien n'est plus loin de ses intentions que d'émettre un jugement. Elle observe seulement un processus soviétique de socialisation qui est de même nature que la formation des mœurs modernes que Norbert Elias a observées sur la longue durée, même si l'auteur ne tente pas de placer son analyse dans cette perspective. Des interactions, interdépendances et interférences finissent assez vite par façonner une intelligentsia soviétique quelque peu éloignée de l'image qu'elle se fait d'elle-même et qu'elle projette.

12 Evgenija Kiseleva, l'auteur du texte maladroit que Paperno compare au journal de Čukovskaja, est l'une de ces créatures que les intellectuels sont enclins à traiter avec un peu de condescendance, surtout à partir du moment où elles empiètent sur leur chasse gardée. Paperno épingle les gardiennes de la pureté des exercices de l'esprit qui ont 
publié l'écrit qu'elles taxent avec paternalisme de « naïf ». Kiseleva a commencé le récit de sa vie pour l'envoyer au studio Mosfilm puisqu'elle considérait celle-ci comme un matériau pour un film. Elle voulait témoigner devant tout le monde, comme Čukovskaja, car elle attachait la même importance historique à son expérience que la compagne d'Ahmatova à la sienne et à celle de la poétesse.

Kiseleva ne mentionne pas la Terreur. Pourtant, son autobiographie commence dans les années 1930. L'événement décisif de son histoire personnelle est la guerre, une catastrophe hors du commun qui n'est qu'une variante de la politique soviétique pour les femmes de lettres. La hantise du souvenir de la guerre fait de Kiseleva une enthousiaste de ce que le régime réussit à vendre comme une politique pacifiste et des champions de cette politique comme Leonid Brežnev. Si bien qu'elle ne mentionne Stalin qu'une seule fois pour blâmer son incapacité à préparer le pays à l'invasion nazie. Elle est même reconnaissante au régime parce qu'il lui garantit une pension modeste qui la rend indépendante de sa famille désintégrée.

Paperno a toutes les raisons de dire que, de prime abord, Čukovskaja, Ahmatova et l'ouvrière vivaient dans deux pays différents. Mais ceci ne l'empêche pas de relever les similarités. Toutes trois se comprennent comme victimes de forces élémentaires qui les dépassent. L'écrivain et l'ouvrière pratiquent une sorte de microhistoire où les événements et les personnages s'insèrent dans un contexte que seul le regard ethnographique peut saisir ; elles décrivent des espaces, des rapports entre des personnes et des sentiments, en principe intimes, où le régime pèse lourd; elles sont parties prenantes de la même culture politique et leur horizon d'attente est un salut que leur univers leur refuse. Paperno montre que la vision de Kiseleva n'est pas plus naïve que la lucidité de membres illustres de l'intelligentsia qui ignorent le monde de l'ouvrière.

Nombre d'écrits intimes d'intellectuels évoquent des rêves dictés par l'angoisse. Bien qu'ils ne soient pas toujours prêts à les interpréter, les dits intellectuels attachent une signification historique et politique aux chimères qui les harcèlent. Il arrive que le rêve prenne une revanche sur les tortionnaires ou que le rêveur continue à vivre le cauchemar de la terreur. Mais il arrive également que des fantasmes soient dictés par un mélange de peur et de sentiment de culpabilité chez ceux qui ont échappé à l'arrestation. L'idée, a priori populaire, que les rêves contiennent des présages est remarquablement tenace même parmi les gens éduqués. Paperno observe que la lecture d'écrits intimes bien avant leur publication dans les milieux intellectuels et les conversations sur toutes sortes de rêves, chimères et peurs contribuaient à renforcer les liens. Le fait de rêver est devenu une sorte d'acte social avec un sous-texte clairement politique.

Paperno conclut que les années de terreur, la peur, la pression du régime, les privations, le danger, la conviction que tout cela créait une communauté et le sentiment qu'il fallait en témoigner, étaient des éléments spécifiquement soviétiques de la socialisation de l'intelligentsia. Leur croisement avec le sentiment traditionnel des intellectuels russes d'être investis d'une mission libératrice formait des personnalités qui voyaient une leçon historique dans leur expérience qu'elles combinaient avec une représentation eschatologique de l'histoire.

Paperno regrette de ne pas être à même de déchiffrer l'univers intérieur de ceux qui n'ont pas écrit de journaux ou de mémoires. On peut la comprendre. Mais on a toutes les raisons de la féliciter de l'extraordinaire travail qu'elle a accompli. 